\title{
Fundamental equations for growth in uniform stands of vegetation
}

\author{
J.L. Monteith* \\ Institute of Terrestrial Ecology, Bush Estate, Penicuik, Midlothian EH26 OQB, UK
}

\begin{abstract}
Applications of the 'expolinear' equation for crop growth described by Goudriaan and Monteith [Goudriaan, J., Monteith, J.L., 1990. Ann. Bot. 66, 695-701] are restricted by the assumption that absolute and specific growth rates are both constant in time. To overcome this constraint, a second-order differential equation is derived that may either be integrated to yield the expolinear equation or used with a daily time-step to simulate growth when absolute and specific growth rates are time-dependent. The expolinear equation fitted the biomass of individual organs in a sorghum trial and related quantities such as leaf area per unit biomass. It predicts that an increase in the variability of weather during early stages of growth will usually reduce biomass production. () 2000 Elsevier Science B.V. All rights reserved.
\end{abstract}

Keywords: Fundamental growth equations; Vegetation; Uniform stands

\section{Introduction}

Many attempts have been made to derive empirical functions for the increase in biomass $(W)$ with time $(t)$ when plants are grown either in isolation or in stands (Thornley and Johnson, 1990). Commonly, these functions incorporate a specific (or relative) growth rate $R$ defined as $(\mathrm{d} W / \mathrm{d} t) / W$ and introduced by Blackman (1919) as the biological equivalent of a financial interest rate. For seedlings able to grow without competition between neighbours, $R$ usually remains effectively constant at a maximum value $R_{\mathrm{m}}$ because $\mathrm{d} W / \mathrm{d} t$ is proportional to the biomass of organs responsible for resource capture which, in turn, is proportional to total biomass. However, $R$ may decline slowly during early growth if there is competition for resources between adjacent organs on the same plant. During the main growth period of an arable crop, $R$ declines steadily because of competition between neighbouring

\footnotetext{
* Tel.: +44-131-445-4343; fax: +44-131-445-3943.
}

plants so that, eventually, the absolute rate of growth $C=\mathrm{d} W / \mathrm{d} t$ becomes almost independent of $W$.

By assuming that the rate of biomass production per unit of intercepted radiation was constant throughout the whole growing season, that irradiance was also constant, and that leaf area was proportional to total biomass, Goudriaan and Monteith (1990) derived an equation for the time-dependence of crop biomass as a function of $R_{\mathrm{m}}$ and of the maximum absolute growth rate $C_{\mathrm{m}}$ commonly observed when there is little change in the availability of resources from week to week. The equation has the form

$$
W(t)=\left(\frac{C_{\mathrm{m}}}{R_{\mathrm{m}}}\right)\left[\ln \left\{1+\left[\frac{f_{0}}{1-f_{0}}\right] \exp \left(R_{\mathrm{m}} t\right)\right\}\right]
$$

where $f_{0}$ is the fraction of incident radiation intercepted by a crop canopy at time $t=0$. Eq. (1) is appropriately described as 'expolinear' because biomass increases exponentially with time when $t$ is small and linearly with time when it is large.

As there are few outdoor environments in which it is legitimate to treat $C_{\mathrm{m}}$ and $R_{\mathrm{m}}$ as independent of 
time over a whole growing season, Eq. (1) is more useful for demonstrating principles than for predicting growth in a specified environment or for analysing field measurements. As shown in the following analysis, however, both $C_{\mathrm{m}}$ and $R_{\mathrm{m}}$ may be treated as being time-dependent when Eq. (1) is replaced by a finite difference equation readily handled within a computer program.

\section{Derivation of a general expolinear equation}

For the short period in which the specific growth rate of a stand may be treated as constant when the environment is constant, the absolute growth rate may be expressed as

$C=\frac{\mathrm{d} W}{\mathrm{~d} t}=R_{\mathrm{m}} W$

Integration with respect to time yields the familiar expression for the exponential increase in biomass with time, viz.

$W=W(0) \exp \left(R_{\mathrm{m}} t\right)$

where $W=W(0)$ when $t=0$. Alternatively, Eq. (2) may be differentiated to obtain the initial 'growth acceleration' as

$\frac{\mathrm{d} C}{\mathrm{~d} t}=R_{\mathrm{m}} C$

Eventually, competition between neighbours limits the absolute growth rate to $C_{\mathrm{m}}$, values of which are set by the availability of resources (water, light, nutrients) per unit land area rather than by physiological demand. To satisfy the final condition that $\mathrm{d} C / \mathrm{d} t=0$ along with the initial condition set by Eq. (4), growth acceleration may be expressed as

$\frac{\mathrm{d} C}{\mathrm{~d} t}=R_{\mathrm{m}} C\left\{1-\frac{C-C_{0}}{C_{\mathrm{m}}-C_{0}}\right\}^{n}$

which is a second-order differential equation in biomass $W$ with $C=C_{0}$ when $t=0$. The index $n$ is introduced to broaden the applicability of Eq. (5) and its impact is demonstrated in Fig. 1 where $W$, obtained by numerical integration of Eq. (5), is plotted as a function of time when $C_{\mathrm{m}}=20 \mathrm{~g} \mathrm{~m}^{-2}$ per day, $C_{0} / C_{\mathrm{m}}=0.003$ and $n$ ranges from 0.5 to 2 . The initial

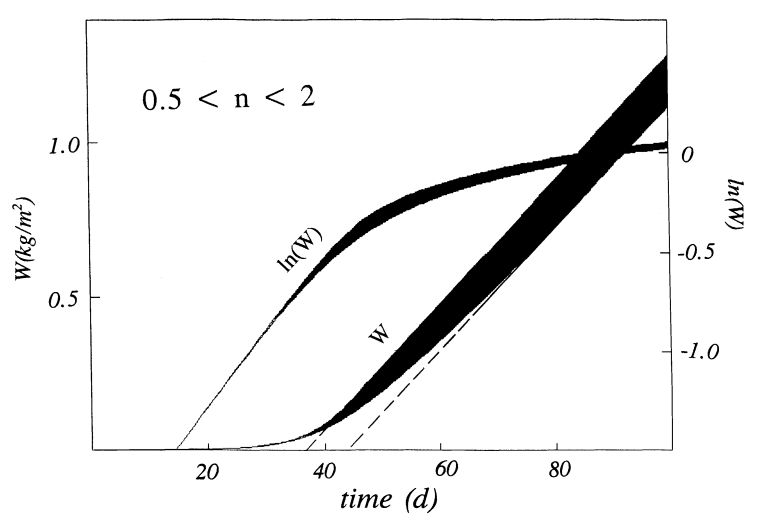

Fig. 1. Increase in biomass $W$ and $\ln (W)$ derived from Eq. (7) with $R_{\mathrm{m}}=0.2$ per day, $C_{\mathrm{m}}=20 \mathrm{~g} \mathrm{~m}^{-2}$ per day, $C_{0} / C_{\mathrm{m}}=0.003$ and $n$ ranging from 2 (lowest limits of $W$ and $\ln (W)$ ) to 0.5 (uppermost limits).

specific growth rate is almost independent of $n$ and the main impact of $n$ on $W$ is through the intercept on the time axis of the linear portion of the relation between $W(t)$ and $t$. This is the time that is 'lost' for resource capture, and therefore, for growth during establishment of the stand. The absolute growth rate approaches $C_{\mathrm{m}}$ about 40 days after emergence (DAE) when $n=0.5$ and after about 60 days when $n=2$, consistent with the fact that many arable crops achieve almost complete ground cover in the second month after emergence.

Eq. (5) derived without reference to a specific process of resource capture can be integrated twice with respect to time to yield an expolinear function similar to Eq. (1). For the special case $n=1$, the first integration yields

$C=\frac{C_{\mathrm{m}} f(t)}{1+f(t)}$

where $f(t)=Q \exp \left(R_{\mathrm{m}} t\right)$ and $Q$ is a constant, evaluated by noting that, when $t=0, C=C_{0}=C_{\mathrm{m}} Q /(1+Q)$. It follows that $Q=C_{0} /\left(C_{\mathrm{m}}-C_{0}\right)\left(=f_{0} /\left(1-f_{0}\right)\right.$ in Eq. (1)), a non-dimensional scale of absolute growth rate effectively equal to $C_{0} / C_{\mathrm{m}}$ when this ratio is very small as in most systems of production.

Eq. (6) may now be integrated to obtain the expolinear equation

$W(t)=\left(\frac{C_{\mathrm{m}}}{R_{\mathrm{m}}}\right)\left[\ln \left(\frac{1+Q \exp \left[R_{\mathrm{m}} t\right]}{1+Q}\right)\right]+W(0)$ 
closely resembling Eq. (1) but with the exact limit of $W(t)=W(0)$ when $t=0$. Commonly, $W(0)$ is much smaller than $W(t)$ for most of the growing season; $Q$ may be neglected in the term $(1+Q)$; and when $t$ is large, $Q \exp \left(R_{\mathrm{m}} t\right)$ is much larger than 1. Eq. (7) may therefore be reduced to

$W(t)=\left(\frac{C_{\mathrm{m}}}{R_{\mathrm{m}}}\right) \ln \left\{1+\exp \left[R_{\mathrm{m}}\left(t-t_{1}\right)\right]\right\}$

where 'lost' time (intercept on the time axis of the linear part of a biomass versus time plot) is given by

$t_{1}=-\frac{\ln (Q)}{R_{\mathrm{m}}}$

As Eq. (8a) (the double integral of Eq. (5) when $n=1$ ) has the same form as Eq. (1) obtained by assuming that the distribution of foliage is random, the parameter $n$ (Eq. (5)) may be regarded as an empirical index of randomness, larger than unity for overdispersed (regularly spaced communities) and smaller for underdispersed (clumped) communities.

\section{Interpretation and extension}

By differentiating Eq. (7) with respect to time, it can be shown that

$C=C_{\mathrm{m}}\left[1-\exp \left\{-\frac{R_{\mathrm{m}}}{C_{\mathrm{m}}} W\right\}\right]$

provided $\exp \left(R_{\mathrm{m}} t\right) \gg 1$ and $W \gg W(0)$. To transform Eq. (9) into a similar and more familiar equation for the relation between growth rate and light interception, $R_{\mathrm{m}}$ is replaced by $C_{0} / W(0)$, leaf area index $L$ is assumed to be proportional to biomass $W$, and a time-independent coefficient for light extinction, $K$, is defined by assuming that $K L_{0}=C_{0} / C_{\mathrm{m}}$ when $t=0$. The exponential term in Eq. (9) then reduces to $\exp (-K L)$ which is the fraction of light transmitted by a leaf area index of $L$. Neglecting the small fraction of light reflected by a canopy, the term in square brackets is therefore equivalent to the fraction of absorbed radiation that Goudriaan and Monteith (1990) assumed to be proportional to growth rate in order to obtain Eq. (1).

Eqs. (4) and (5) were derived by assuming that the growth of plants in a stand is limited initially by plant size, and therefore, by biomass, but eventually becomes limited by the availability of resources in the environment. A similar distinction can be made between the relatively slow initial growth of a specific organ (leaf, stem, root) which is likely to depend on its biomass (or 'sink strength'), and a maximum final rate of growth limited by the availability of assimilate (or 'source strength'). The equations may therefore have limited validity for organs as well as more general validity for plant stands.

If other attributes of growth such as leaf area were to be expolinear functions of time, it would be possible to establish compound quantities such as specific leaf area or leaf area per unit total biomass by dividing one expolinear equation by another. Such quantities will be time-dependent unless values of $R_{\mathrm{m}}$ and $C_{0} / C_{\mathrm{m}}$ are identical for both processes (see below) so that 'lost' times are also identical.

\section{Non-dimensional expolinear equation}

To compare the growth of different species in the same environment or of different organs on plants within the same stand, it is convenient to write Eq. (8a) in the non-dimensional form

$$
W^{*}(t)=\ln \left\{1+\exp \left(t^{*}\right)\right\}
$$

where $W^{*}(t)=\{W(t)-W(0)\}\left(R_{\mathrm{m}} / C_{\mathrm{m}}\right)$ is a non- dimensional mass and $t^{*}=R_{\mathrm{m}}\left(t-t_{1}\right)$ a non-dimensional time. As measurements of biomass in a stand are commonly deferred until $W^{*}(t)$ is much larger than $W^{*}(0)$, the latter term can usually be neglected.

During the initial stages of growth when $\exp \left(t^{*}\right)$ is much less than 1, Eq. (8a) assumes a lower limit of $W^{*}=\exp \left(t^{*}\right)$, implying that biomass increases exponentially with time. If $t^{*}$ increases until $\exp \left(t^{*}\right)$ is much larger than 1 , the equation has an upper limit $W^{*}=t^{*}$ so that biomass increases linearly with time.

\section{Field tests in a quasi-constant environment}

Eqs. (6)-(9) describe the growth of a plant stand in a constant environment of the type available in a phytotron. In most climates, however, at least one significant element, usually daily mean temperature or radiation, changes significantly during the growing season. Exceptionally, at the beginning of the 


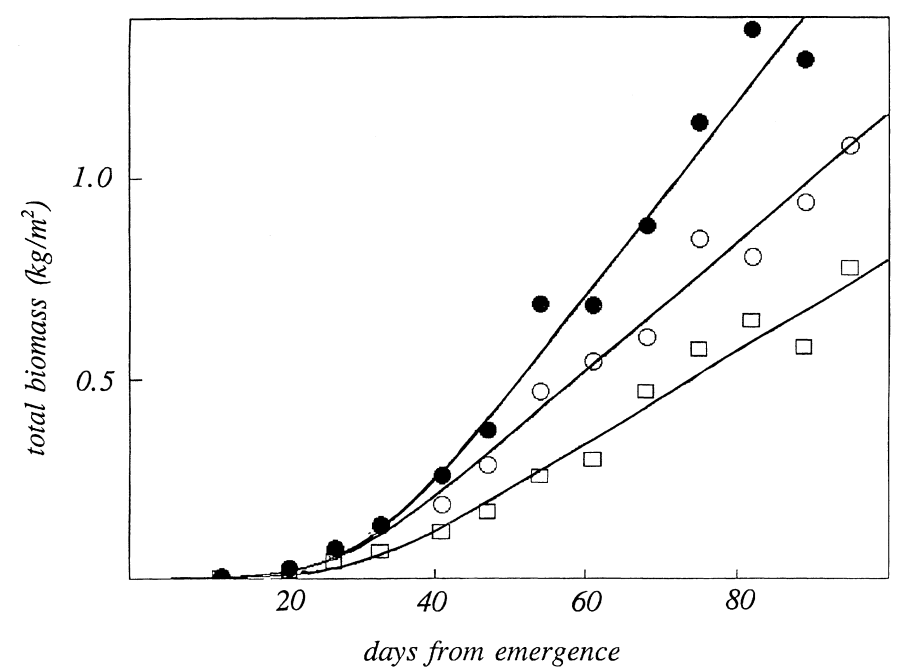

Fig. 2. Increase in total biomass of sorghum grown during rainless weather at ICRISAT, Hyderabad, India. ( $\square$ ) No irrigation or applied $\mathrm{N}$; (O) no irrigation and $150 \mathrm{~kg} \mathrm{~N} / \mathrm{ha}$; (O) irrigation and $150 \mathrm{~kg} \mathrm{~N} / \mathrm{ha}$.

dry season in the semi-arid tropics, crops grow on stored water during a rainless period when the sky is usually cloudless so that radiation is uniform and mean temperature declines only slowly with time. Observations on the growth of sorghum in such an environment have been reported by Rego et al. (1998) and by Singh et al. (1998) working at the International Crops Research Institute for the Semi-Arid Tropics (ICRISAT), Hyderabad. They grew a sorghum cultivar (SPH-260) on a deep Vertisol at six levels of fertilization $(0-150 \mathrm{~kg} \mathrm{~N} / \mathrm{ha})$ and at two levels of irrigation (zero after initial wetting throughout the profile and restoration of the profile to field capacity approximately every 2 weeks from emergence until 50 days later). Three replicate samples were obtained from all treatments, weekly for leaves and stems and every 2 weeks for roots.

In Fig. 2, total biomass is plotted as a function of time for three contrasting treatments. Expolinear equations (parameters in the legend of Fig. 3) fit the data well and illustrate an important feature: $C_{\mathrm{m}}$ doubled in response to the application of water and nitrogen. In contrast, $R_{\mathrm{m}}$ and $C_{0} / C_{\mathrm{m}}$ were effectively independent of treatment, implying that $t_{1}$ (Eq. (8b)) was also independent of treatment. Therefore, measurements for all treatments fall on the universal growth curve obtained by plotting the normalized biomass $W R_{\mathrm{m}} / C_{\mathrm{m}}$ against normalized time $R_{\mathrm{m}}\left(t-t_{1}\right)$ (Fig. 3).
In this trial, the biomass of individual organs also increased with time in an expolinear fashion. However, the growth of leaves (Fig. 4) stopped abruptly at about $t^{*}=8$ corresponding to $60 \mathrm{DAE}$ when anthesis was recorded. In contrast, stems and roots continued to grow until physiological maturity at about $t^{*}=17$ corresponding to 90-95 DAE.

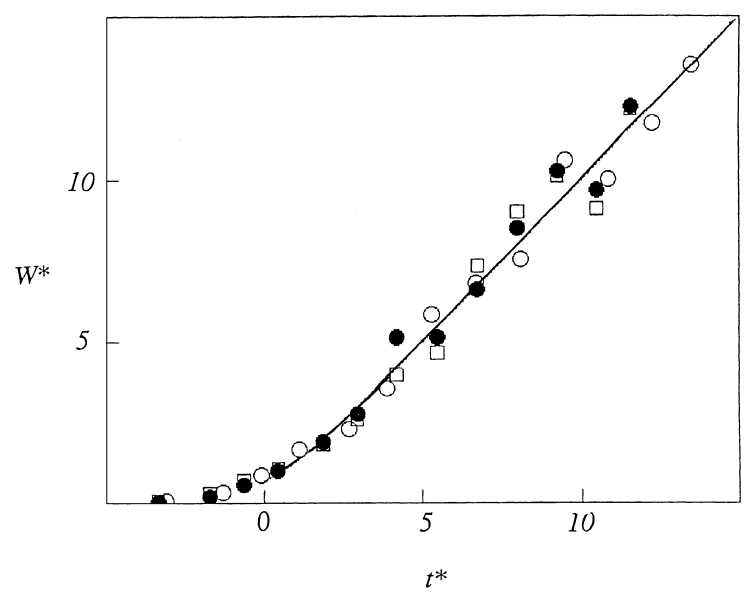

Fig. 3. Non-dimensional plot of total biomass $\left(W^{*}=W R_{\mathrm{m}} / C_{\mathrm{m}}\right)$ for sorghum as a function of non-dimensional time from emergence $\left(t^{*}=R_{\mathrm{m}}\left[t-t_{1}\right]\right)$ obtained from the data in Fig. 2 assuming identical initial conditions for all three treatments, viz. $R_{\mathrm{m}}=0.19$ per day, $C_{0} / C_{\mathrm{m}}=0.004, t_{\mathrm{l}}=29$ days after emergence (DAE). Values of $C_{\mathrm{m}}$ (in $\mathrm{g} \mathrm{m}^{-2}$ per day) are $11.5(\square), 16.0(\bigcirc)$ and $24.0(\mathbf{O})$. 


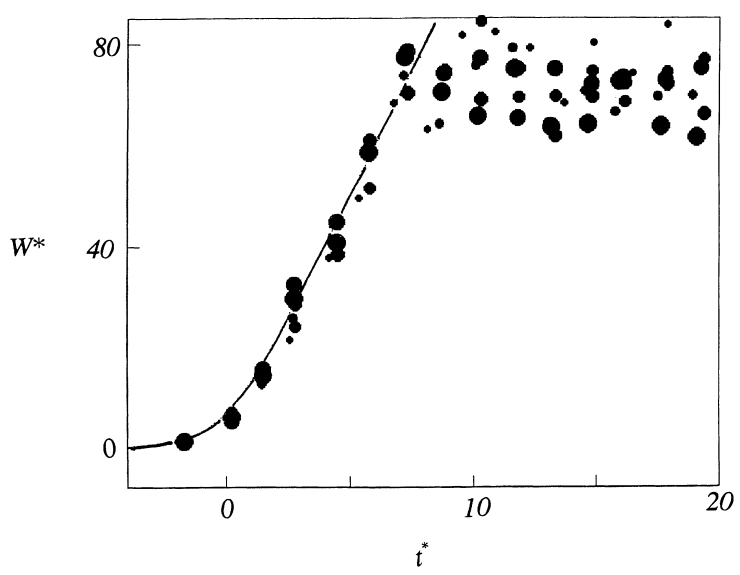

Fig. 4. Non-dimensional plot of biomass for leaves of sorghum grown at six rates of nitrogen $(0,30,60,90,120,150 \mathrm{~kg} / \mathrm{ha})$ ranked by size of the symbol.

The expolinear increase in biomass with time was accompanied by an expolinear increase in leaf area until anthesis. Thereafter, leaf area decreased with time at an approximately constant rate. Fig. 5 illustrates this behaviour for a treatment ( $150 \mathrm{~kg} \mathrm{~N} / \mathrm{ha}$ with irrigation) in which the 'lost' time for leaf area was 32 DAE compared to 45 days for total biomass. In consequence, the leaf area per unit biomass increased rapidly until about $30 \mathrm{DAE}$ and then decreased equally rapidly until maturity at about 100 DAE. The curve representing leaf area index per unit biomass was obtained in three stages: (a) fitting leaf area to an expolinear equation up to $60 \mathrm{DAE}$ and to a linear equation for the subsequent senescent phase; (b) fitting total biomass to a second expolinear equation; (c) dividing the output from (a) by the output from (b). A general inference from this analysis is that relatively small differences in lost time for two components of growth can be responsible for a more complex response to time in the ratio of these components. Interpretation of growth in such terms has potential for revealing substantially more information about underlying processes than the traditional fitting of arbitrary empirical functions.

At least in a quasi-constant environment, it appears that the expolinear equation may be applicable to the growth of individual organs as well as to ontogenetic changes of compound quantities such as leaf biomass or nitrogen content per unit leaf area. The procedure to be followed in a variable environment is discussed in Section 6.

\section{Yield and weather variability}

The possibility that crop yields may depend on the variability of weather as well as on average conditions during the growing season has been suggested by field observations and appears to be consistent with the evidence from models in which variability has been simulated (e.g. by Semenov and Porter, 1995). As the models are complex, the source of this

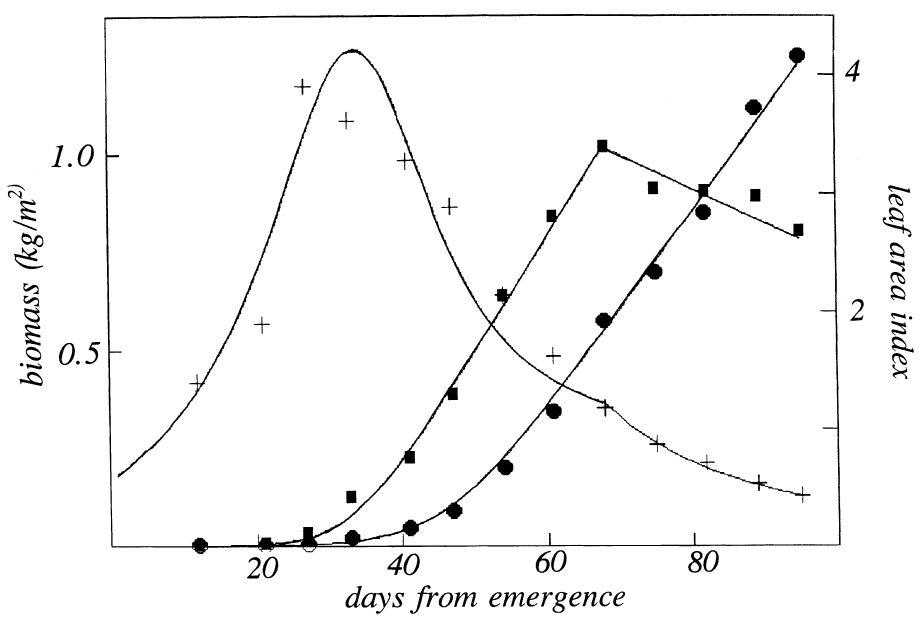

Fig. 5. Leaf biomass $(\mathbf{)})$, leaf area $(\mathbf{\square})$ and leaf area ratio (+) for sorghum (see text). 


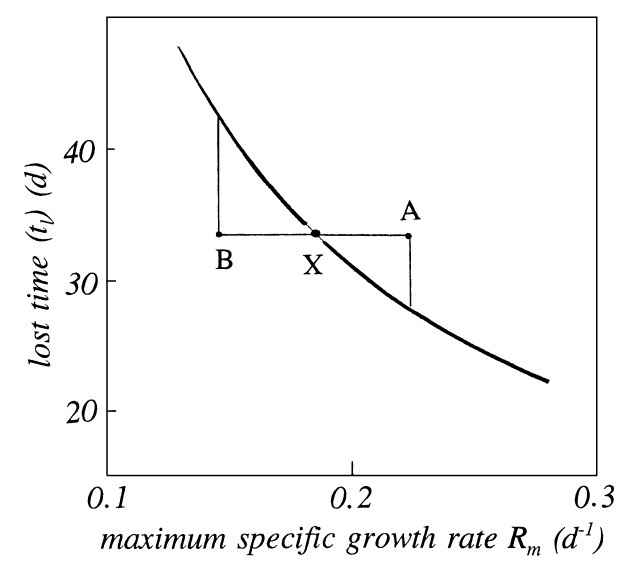

Fig. 6. Dependence of lost time $t_{1}$ on specific growth rate $R_{\mathrm{m}}$ when $C_{0} / C_{\mathrm{m}}=0.004$.

dependence has not been explicit, but the dependence of 'lost' time on the maximum specific growth rate $R_{\mathrm{m}}$ may be a significant factor.

Laboratory evidence suggests that $R_{\mathrm{m}}$ may be assumed to increase linearly with temperature at least over a narrow range. As $\mathrm{d} t_{1} / \mathrm{d} R_{\mathrm{m}}$ is negative but $\mathrm{d}^{2} t_{1} / \mathrm{d} R_{\mathrm{m}}^{2}$ is positive (Fig. 6), any increase in $R_{\mathrm{m}}$ (e.g. from $X$ to $A$ ) as a result of an increase in temperature, say, will be responsible for a decrease in $t_{1}$ smaller than the complementary increase in $t_{1}$ (from $X$ to $B$ ) induced by a negative temperature fluctuation of identical magnitude. It follows that, during the establishment of a stand when growth is strongly dependent on the magnitude of $R_{\mathrm{m}}$, fluctuations in an environmental factor such as air temperature causing $R_{\mathrm{m}}$ to fluctuate will result in a loss of production, albeit small in many circumstances. In the main period of growth, however, where the growth rate is dominated by the value of $C_{\mathrm{m}}$, production (at a rate proportional to $C_{\mathrm{m}}$ ) should not be affected by small fluctuations in the growth rate about its mean value.

Eq. (4) has the important property that it defines the relation between $\mathrm{d}^{2} W / \mathrm{d} t^{2}$ and $\mathrm{d} W / \mathrm{d} t$ in terms of initial relative and final absolute growth rates but not in terms of the initial biomass or the initial absolute growth rate. It is therefore possible to use the equation to obtain the increase in growth rate and biomass with time numerically, adopting, for example, a daily time-step and values of $R_{\mathrm{m}}$ and $C_{\mathrm{m}}$ that depend on the state of the environment, and therefore, on time. If the time step is $\delta t$, the crop growth rate $C(t)(=\mathrm{d} W / \mathrm{d} t)$ on Day $t$ is given by

$C(t+\delta t)=C(t)+\delta t \mathrm{~d} C(t) / \mathrm{d} t$

where, from Eq. (5) (neglecting the small term $C_{0}$ ),

$\frac{\mathrm{d} C(t)}{\mathrm{d} t}=C(t) R_{\mathrm{m}}(t)\left\{1-\frac{C(t)}{C_{\mathrm{m}}(t)}\right\}$

Biomass at the start of Day $t+1$ is then evaluated as

$W(t)=W(t-\delta t)+\delta t C(t)$

This behaviour is illustrated in Fig. 7 where the circles represent biomass estimated as a function of time using Eq. (7) with constant values of $R_{\mathrm{m}}$ ( 0.2 per day) and of $C_{\mathrm{m}}\left(10 \mathrm{~g} \mathrm{~m}^{-2}\right.$ per day for $\mathrm{C} 3$ species - open circles; and $20 \mathrm{~g} \mathrm{~m}^{-2}$ per day for $\mathrm{C} 4$ species — full circles). The two shaded portions were obtained with the same values of $C_{\mathrm{m}}$ but with environmental fluctuations introduced by assigning random daily values between 0.18 and 0.22 per day to $R_{\mathrm{m}}$. In practice, fluctuations of this order could be a consequence of daily mean temperature fluctuating by $\pm 2{ }^{\circ} \mathrm{C}$ about a mean of $20^{\circ} \mathrm{C}$, assuming that the relative growth rate is proportional to temperature over the range considered.

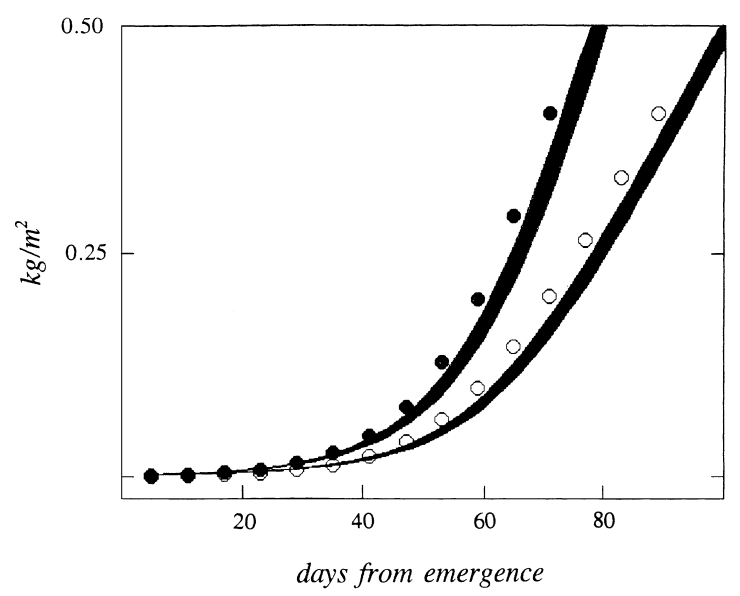

Fig. 7. Estimated increase in biomass with time assuming constant values of $C_{\mathrm{m}}$, viz. $10 \mathrm{~g} \mathrm{~m}^{-2}$ per day (○) or $20 \mathrm{~g} \mathrm{~m}^{-2}$ per day (O). Shaded areas obtained by allowing $R_{\mathrm{m}}$ to assume daily values between 0.18 and 0.22 per day. 


\section{Conclusions}

The differential equation (Eq. (5)) that forms the basis of the expolinear equation (Eq. (7)) provides a new method for simulating the growth of crop stands and possibly of their components. Unlike more complex systems of simulation, it is based on a secure foundation of calculus and operates with a minimum of parameters and arbitrary constants. The main problem in using the equation practically is common to all types of modelling which assume values for the dependence of dominant parameters $\left(C_{\mathrm{m}}, R_{\mathrm{m}}\right.$ and $Q$ in this paper) on physiology, management, and the state of the environment both above and below the ground.

\section{References}

Blackman, V.H., 1919. The compound interest law of plant growth. Ann. Bot. 30, 353-360.

Goudriaan, J., Monteith, J.L., 1990. A mathematical function for crop growth. Ann. Bot. 66, 695-701.

Rego, T.J., Monteith, J.L., Singh, P., Lee, K.K., Nageswara Rao, V., Srirama, Y.V., 1998. Response to fertiliser nitrogen and water of post-rainy season sorghum on a Vertisol. 1. Biomass and light interception. J. Agric. Sci. 131, 417-428.

Semenov, M.A., Porter, J.R., 1995. Climatic variability and the modelling of crop yields. Agric. For. Meteorol. 73, 265-283.

Singh, P., Monteith, J.L., Lee, K.K., Rego, T.J., Wani, S.P., 1998 Response to fertiliser nitrogen and water of post-rainy season sorghum on a Vertisol. 2. Biomass and water extraction. J. Agric. Sci. 131, 429-438.

Thornley, J.T., Johnson, I.R., 1990. Plant and Crop Modelling. Clarendon Press, Oxford. 